\title{
Real-time in vivo monitoring of magnetic nanoparticles in the bloodstream by $A C$ biosusceptometry
}

\author{
André G. Próspero ${ }^{1}$, Caio C. Quini ${ }^{1}$, Andris F. Bakuzis ${ }^{2,4^{*}}{ }^{\mathbb{D}}$, Patrícia Fidelis-de-Oliveira' ${ }^{1}$, Gustavo M. Moretto ${ }^{1}$, \\ Fábio P. F. Mello ${ }^{1}$, Marcos F. F. Calabresi', Ronaldo V. R. Matos' ${ }^{1}$, Ednaldo A. Zandoná ${ }^{1}$, Nícholas Zufelato², \\ Ricardo B. Oliveira ${ }^{3}$ and José R. A. Miranda'
}

\begin{abstract}
Background: We introduce and demonstrate that the AC biosusceptometry (ACB) technique enables real-time monitoring of magnetic nanoparticles (MNPs) in the bloodstream. We present an ACB system as a simple, portable, versatile, non-invasive, and accessible tool to study pharmacokinetic parameters of MNPs, such as circulation time, in real time. We synthesized and monitored manganese doped iron oxide nanoparticles in the bloodstream of Wistar rats using two different injection protocols. Aiming towards a translational approach, we also simultaneously evaluated cardiovascular parameters, including mean arterial pressure, heart rate, and episodes of arrhythmia in order to secure the well-being of all animals.
\end{abstract}

Results: We found that serial injections increased the circulation time compared with single injections. Immediately after each injection, we observed a transitory drop in arterial pressure, a small drop in heart rate, and no episodes of arrhythmia. Although some cardiovascular effects were observed, they were transitory and easily recovered in both protocols.

Conclusions: These results indicate that the ACB system may be a valuable tool for in vivo, real-time MNP monitoring that allows associations with other techniques, such as pulsatile arterial pressure and electrocardiogram recordings, helping ensuring the protocol safety, which is a fundamental step towards clinical applications.

Keywords: AC biosusceptometry, Magnetic nanoparticles, Circulation time, Pharmacokinetics, Cardiovascular analysis

\section{Background}

Magnetic nanoparticles (MNPs) are known for their wide versatility in several applications fields [1]. Specifically regarding biomedical applications, they have been employed on cell detection and separation [2], stem cell tracking and signaling [3, 4] and also as therapeutic agents for hyperthermia [5-7] and drug delivery [8]. This broad range of possibilities is a unique factor that allows their application on both diagnosis and treatment, leading to multimodal applications $[9,10]$.

*Correspondence: abakuzis@ufg.br; bakuzis@ufg.br

${ }^{4}$ Instituto de Física-Universidade Federal de Goiás, Goiânia, GO

74690-900, Brazil

Full list of author information is available at the end of the article
Preclinical characterization of nanoparticles is a crucial step towards clinical applications and, therefore, has garnered great interest and efforts from the scientific community $[11,12]$. The circulation time, or half-life $\left(\mathrm{T}_{1 / 2}\right)$, of nanostructured agents is a parameter of major relevance for in vivo experimentation and clinical procedures $[8,9$, 13, 14]. Also, monitoring biodistribution, and clearance under in vivo conditions is of paramount importance in the field [15]. All these properties are mainly controlled by nanoparticles intrinsic properties, such as superficial charge, coating material, core and size, which influence directly both circulation time and the target region [11, 15]. 
Pharmacokinetic modeling is the current gold standard method to assess $T_{1 / 2}$ of nanoparticles. This approach is based upon sequential measurements of MNPs blood concentrations (i.e., ex vivo studies) [16]. Although several techniques have been employed for such purposes, such as electron spin resonance (ESR) $[17,18]$ or inductively coupled plasma mass spectrometry (ICP-MS) [19, 20], this method can only provide snapshots at specific time points.

Thus, developing proper detection methods that allow for in vivo studies and real-time monitoring is crucial in order to improve nanoparticles applicability and to enable real translational approaches [13, 21].

Among the current techniques, based on direct measurements that allow in vivo particle detection, are magnetic resonance imaging (MRI) [13] and magnetic particle imaging (MPI) [22]. MRI presents some limitations, specially distinguishing particle location within tissue with low signal [23]. On other hand, the MPI system has shown great promise for nanoparticle detection and has already been reported to be able to track stem cells, within a 200 cells threshold (i.e., two orders of magnitude lower than the number of cells that can be detected by MRI) [23]. However, the use of MPI is currently limited to only a few scientists because of the complexity and high cost associated. Thus, there is still an urgent need to develop new simple and accessible techniques that can provide real-time information on MNP availability for in vivo applications.

The AC biosusceptometry (ACB) system is a biomagnetic technique, already described and employed on pharmacological and gastrointestinal studies, in both animals and humans [24-26], and recently employed on magnetic nanoparticles detection [27]. When compared with similar detection systems, ACB is an accessible, versatile, radiation-free and non-invasive technique with unique temporal resolution.

Another aspect that has drawn attention with regard to intravenous nanoparticle applications is the physiological effect of the administration protocol. The cardiovascular effects of intravenous MNP administration have been investigated $[28,29]$.

In order to achieve a real approach considering future clinical applications, it is necessary to find detection technologies that enable the association with standard methods to monitor physiological and, specifically, cardiovascular parameters during the experimental procedure [30]. However, to the best of our knowledge, there are no studies focused on nanoparticle circulation time and the possible effects of MNP intravenous injections on cardiovascular parameters, simultaneously.

Thus, the purpose of our study was to apply the ACB device to monitor MNPs in the bloodstream, in vivo and in real-time, for two different administrations protocols, with simultaneous assessments on potential cardiovascular effects, ensuring the safety of the procedure and decreasing the time and the number of animals employed in this kind of studies.

\section{Methods}

We performed a simultaneous, real-time evaluation of circulation time and cardiovascular parameters after intravenous injections of MNPs. We employed an ACB system to assess the circulation time of nanoparticles in the bloodstream and how it changes for different injections protocols. We simultaneously monitored mean arterial pressure (MAP), heart rate (HR), and cardiac electrical profile with the objective of ensuring the procedure safety and also guaranteeing that all changes in circulation time and nanoparticles availability were due to modifications in the injection protocol.

\section{Magnetic nanoparticles}

We employed a citrate coated, manganese ferrite nanoparticle $\left(\mathrm{Cit}-\mathrm{MnFe}_{2} \mathrm{O}_{4}\right.$ ), with physical core diameter of $15 \pm 5 \mathrm{~nm}$, hydrodynamic diameter of $51.2 \mathrm{~nm}$, superficial charge of $-27.8 \mathrm{mV}$ and polydispersion index of the colloid sample of 0.21 in the stock solution with concentration of $45 \mathrm{mg} / \mathrm{ml}$. The Fe and $\mathrm{Mn}$ content were found to be $74.4 \pm 2.6$ and $25.6 \pm 2.6 \%$, respectively. The 3:1 ratio is related to a passivation process (see Additional file 1) that enriches the nanoparticle surface with Fe. The Cit-MNP was synthesized by co-precipitation method $[5,31]$ and presented a saturation magnetization of $49.4 \mathrm{emu} / \mathrm{g}\left(247 \mathrm{emu} / \mathrm{cm}^{3}\right)$, showing a quasi-static superparamagnetic behavior (i.e., the nanostructure did not present any coercive field under direct current (DC) conditions). The MNPs presented a hydrodynamic size increase when in contact with biological media (reaching $110 \mathrm{~nm}$ approximately). All information concerning the nanoparticles synthesis and its physical and biological characterization processes are described in the Additional file 1 (sections 1, 2).

\section{AC biosusceptometry}

The ACB system is a magnetic material detector that works as a double magnetic flux transformer, wherein the excitation/detection coil pair, farther from the magnetic material, acts as a reference (i.e. first order gradiometer configuration). When there is no magnetic material close to the measurement system, the signal response is minimized. By closing the gap between the magnetic material and detection pair, an imbalance occurs in the magnetic flux, increasing the electrical signal acquired. This electrical signal can be measured, digitized, and recorded online with the assistance of a sensitive-to-phase 
amplifier (lock-in), analog/digital card, and a computer. The description and characterization of the system are detailed in the Additional file 1 (sections 3, 4).

\section{Animal experimentation}

We used 12 male rats (Rattus norvegicus albinus, Wistar), provided by Biotério Anilab (Paulínia, SP, Brazil), weighing 250-300 g. The animals were selected and maintained under suitable conditions with ad libitum feeding (ethics protocol CEUA-IBB 649). All of the animals underwent intraperitoneal urethane anesthesia $(1.5 \mathrm{mg} / \mathrm{kg})$, followed by femoral vein and artery cannulation, for intravenous MNPs administration and pulsatile arterial pressure (PAP) acquisition, respectively. For electrocardiography (ECG), the electrodes were inserted in the D2 derivation, and the ACB sensor was positioned over the animal's cardiac projection (Fig. 1).

Each animal received one or more doses of Cit-MNP, at a rate of $30 \mu \mathrm{l} / \mathrm{s}$, according to their respective experimental group: G1 (six animals received three injections of $300 \mu \mathrm{l}$ of Cit-MNPs at $35 \mathrm{~min}$ intervals) and G2 (six animals received only one injection of $900 \mu \mathrm{l}$ of Cit-MNPs.)

After MNP administration and online data acquisition (ACB, ECG, and PAP), we killed all of the animals by decapitation while they were still under anesthesia (90 min after the first MNP administration).

\section{Acquisition and quantification}

We performed online acquisition using a Lock-in amplifier and Biopac system at a sampling rate of $200 \mathrm{~Hz}$ for all magnetic and electrical signals.

We assessed the pharmacokinetics parameters (i.e., MNP circulation time) employing two different methods:

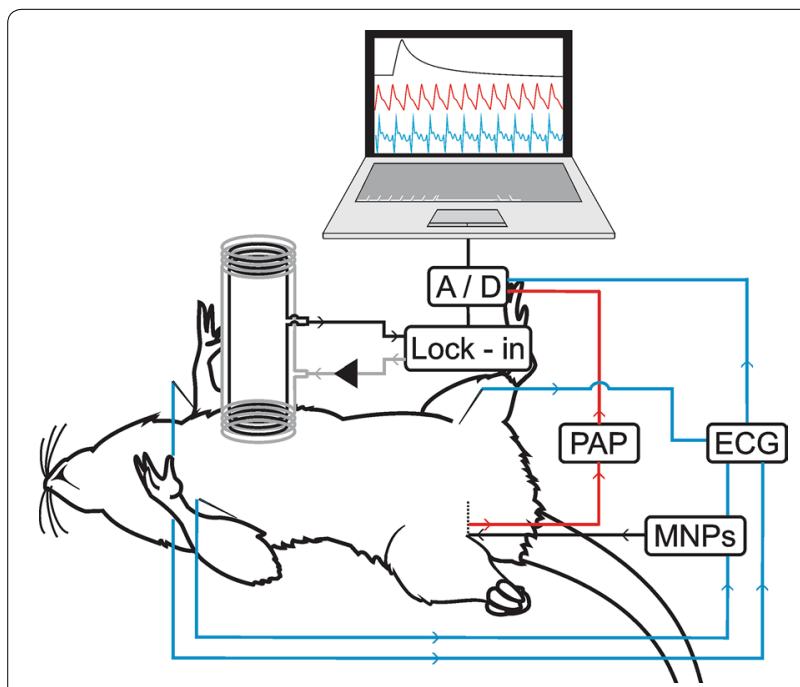

Fig. 1 Experimental setup for MNP administration and data acquisition
The $\mathrm{T}_{1 / 2}$ (half-life time) and the Mean Residence Time (MRT). To study the circulation time in each injection performed in G1 and in the sole injection in G2, we assess the circulation time in each animal by a single exponential decay and quantified it by a single-phase $T_{1 / 2}$ fitting model $[8,20,32]$. However, a $\mathrm{T}_{1 / 2}$ corresponding to the entire circulation time in G1 cannot be calculated by a single-phase fitting model, due to the signals' shape (three peaks and their decays along signal). Thus, in order to compare both administration protocols, we employed a statistical moment approach. This strategy resulted in a MRT for each animal, which is calculated by the temporal parameter weighted by the ACB intensity curve and normalized by the area under the curve. In this way, the MRT calculation takes into account the entire signal, regarding the three injections for G1 (three injections at a 35 min interval between them, with total data acquisition period of $105 \mathrm{~min}$ ), and one single injection for G2 (also with a 105 min of acquisition interval). The MRT calculation is a well described method widely used to assess the pharmacokinetics regarding multiple dosing regimens [33-35], and to quantify gastric emptying and gastrointestinal transit time $[27,36]$. The MRT can be obtained according to Podczeck et al. [36]:

$$
M R T=\frac{\int_{0}^{t_{\max }} t \cdot I_{t} d t}{\int_{0}^{t_{\max }} I_{t} d t}
$$

where $I_{t}=\mathrm{ACB}$ intensity signal at time t.

We also quantified the ACB signal intensity increase $\left(\mathrm{I}_{\mathrm{INC}}\right)$ after each injection performed in G1 and the injection in G2. To study the ACB reproducibility we summed all three $\mathrm{I}_{\mathrm{INC}}$ values obtained from $\mathrm{G} 1$ and compared with the $\mathrm{I}_{\mathrm{INC}}$ obtained in G2. The overall maximum intensity signal reached in each animal $\left(\mathrm{I}_{\mathrm{MAX}}\right)$, was also quantified. We also measured the MNP arrival time $\left(\mathrm{T}_{\mathrm{A}}\right)$ in the heart after systemic injections in both groups. $\mathrm{T}_{\mathrm{A}}$ is the time from the injection start until the signal reaches the $\mathrm{I}_{\text {INC }}$ (e.g., its highest ACB signal intensity after each injection). See Additional file 1 for further details of the quantification process (sections 4, 5).

For the cardiovascular analysis, we analyzed the RR interval (i.e., the regularity of repetitions of $R$ waves on the electrocardiogram) to observe the possible presence of arrhythmias in the cardiac electrical profile of after intravenous MNP administration. We also assessed the effects of intravenous MNP administration on mean arterial pressure (MAP), heart rate (HR), which was calculated based on the PAP signal obtained. From the PAP signal, we also quantified the maximum hypotension instant (MHI) and the MAP recovery time, which is, respectively, the instant at which arterial pressure reached a minimum value and the necessary time for the 
arterial pressure to return to baseline values. PAP, ECG, and $\mathrm{ACB}$ signals were registered and quantified using Aknowlodge 4.1.1, Matlab 2011and OriginPro 8 software.

\section{Statistical analysis}

All of the data was expressed as mean \pm standard deviation. For analyses of specific parameters under two different conditions in the same animal, we applied paired Student's $t$ tests. We used unpaired Student's $t$ test when we analyzed the same parameter in different animals. When evaluating a specific parameter that involved the same animals under three different conditions, we used one-way repeated-measures analysis of variance, followed by the Tukey post hoc test for comparisons between groups. Values of $p<0.05$ were considered statistically significant.

\section{Results}

\section{Magnetic nanoparticle monitoring by ACB system}

We found a similar curve profile of the magnetic intensity overtime in all of the animals after the MNP injections. Figure 2A shows a representative signal acquired in the G1 group, illustrating the magnetic peak intensity after each injection $\left(\mathrm{I}_{\mathrm{INC}}\right)$. Each signal showed a sharp peak immediately after the MNP injection, followed by exponential decay, which could be associated with the particles arriving in the heart and clearance from the bloodstream, respectively. Figure 2B shows a representative signal acquired in the G2 group, illustrating the fitting curve that was applied to all of the data. Comparisons of each injection curve profile in the G1 group are illustrated in Fig. 2C. Note that the graphic scales, in both Fig. 2B, C, are different, wherein the intensities for G1 group are considerably lower than in G2.

Figure 2 also describes how sequential particle administrations influenced the signal profile, in which the second and third peaks resulted in a lower rate of signal decay, suggesting a reduction of the nanoparticles' uptake pattern and a consequent increase in $\mathrm{T}_{1 / 2}$. Multiple injections also influenced the amount of circulating particles (residual intensity $\left[\mathrm{I}_{R}\right]$ ), indicating higher MNP availability in the bloodstream after the latter injections.

Figure 3 presents the ACB signal intensity increase $\left(\mathrm{I}_{\mathrm{INC}}\right)$ detected in the G1 group after each MNP
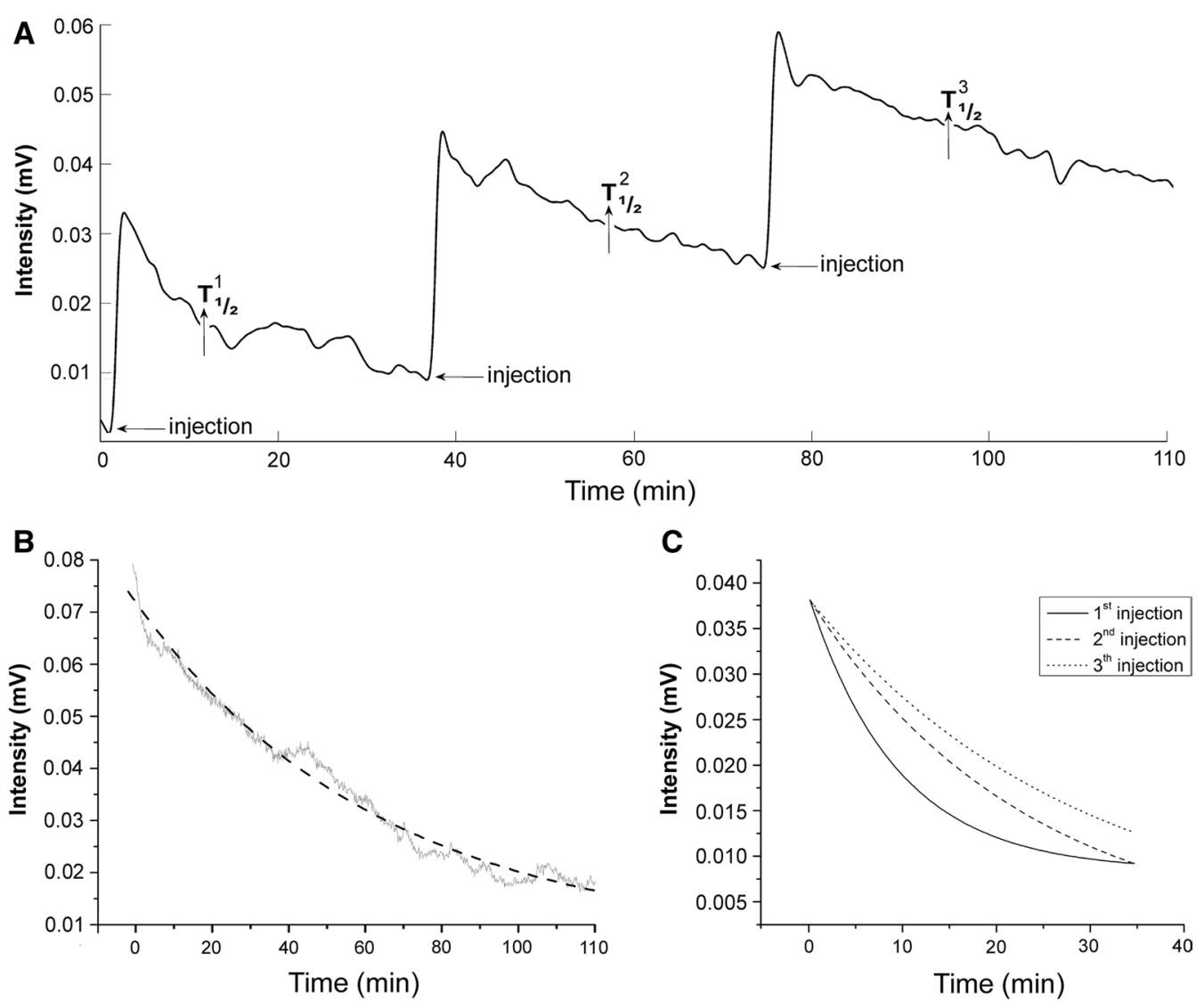

Fig. 2 Magnetic signal acquired by the ACB system. A Example of signal in the G1 group. B Signal acquired and fitting applied in the G2 group. C Fitting curves from each injection performed in the G1 group 
injection was not significantly different between injections (Fig. 3A). When we summed the intensity increase observed in all three injections and compared it with the results from the G2 protocol, the $\mathrm{I}_{\mathrm{INC}}$ were not significantly different between groups, confirming that the $\mathrm{ACB}$ system detected a similar signal intensity increase when
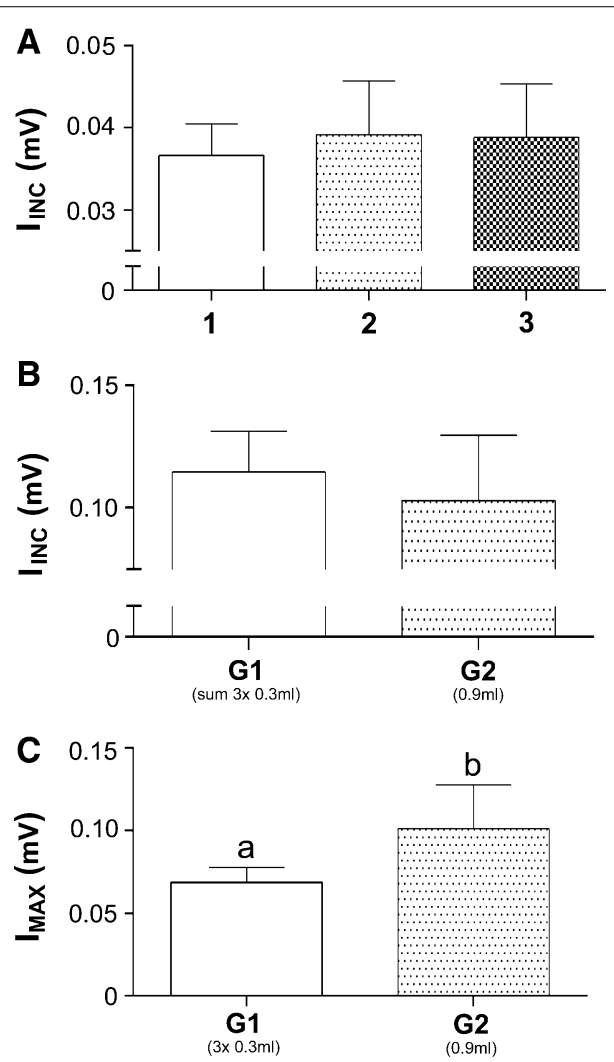

Fig. 3 A $\left.\right|_{\mathbb{N} C}$ detected for each injection in G1. B Comparison of sum of $I_{I_{N C}}$ in $G 1$ and $I_{I_{N C}}$ in G2. C Comparison of $I_{\text {MAX }}$ reached in each group. Different letters indicate significant differences between groups $(p<0.05)$ the particles concentration was the same, even after different injection protocols (Fig. 3B).

Figure $3 \mathrm{C}$ shows the $\mathrm{I}_{\mathrm{MAX}}$ reached in both protocols. In $\mathrm{G} 1$ group the maximum intensity was lower than the $\mathrm{I}_{\mathrm{MAX}}$ reached in G2, which was an expected outcome, since this protocol consisted of sequential injections, giving sufficient time for uptake mechanisms to remove part of the MNP from the circulation before its final injection, and consequential maximum concentration in the bloodstream $\left(\mathrm{I}_{\text {MAX }}\right)$, could be reached.

On regards to circulation time, the $T_{1 / 2}$ significantly increased when we compared all injections (Fig. 4A). These results presented a linear trend $\left(\mathrm{R}^{2}=0.78\right.$, $p=0.0001)$, confirming a relationship between sequential injections and $\mathrm{T}_{1 / 2}$, in which this parameter increased proportionally to each injection.

We found a $\mathrm{T}_{1 / 2}$ of $11.5 \pm 3.5 \mathrm{~min}, 20.3 \pm 2.1 \mathrm{~min}$, and $24.7 \pm 3.9 \mathrm{~min}$ for the first, second, and third injections, respectively, in the G1 group (Fig. $4 \mathrm{~A}$ ). The $\mathrm{T}_{1 / 2}$ obtained for the single administration in $\mathrm{G} 2$ group was $46.7 \pm 4.3 \mathrm{~min}$, which was statistically different from each administration in G1 group. Regarding circulation time for the entire signal acquired, the comparison between protocols was performed over MRT calculations. We found a MRT of $64.0 \pm 1.4$ and $47.1 \pm 3.6 \mathrm{~min}$ for the G1 and G2 groups, respectively (Fig. 4B), indicating a longer circulation time in multiple-dosing regimen compared with one sole injection.

The average $\mathrm{T}_{\mathrm{A}}$ for each administration in the G1 group was approximately $20 \mathrm{~s}$, while the $T_{A}$ for $G 2$ was $52 \mathrm{~s}$, as shown in Table 1. Thus, in the G1 group, the $300 \mu \mathrm{l}$ volume was injected after $10 \mathrm{~s}$ from the procedure start, taking approximately, another $10 \mathrm{~s}$ to reach the maximum signal increase. Since the injection rate was the same for both groups ( $30 \mu \mathrm{l} / \mathrm{s})$, in G2 the $900 \mu \mathrm{l}$ volume was completely injected after $30 \mathrm{~s}$ from the start, taking another $20 \mathrm{~s}$ to reach the maximum signal increase. This delay
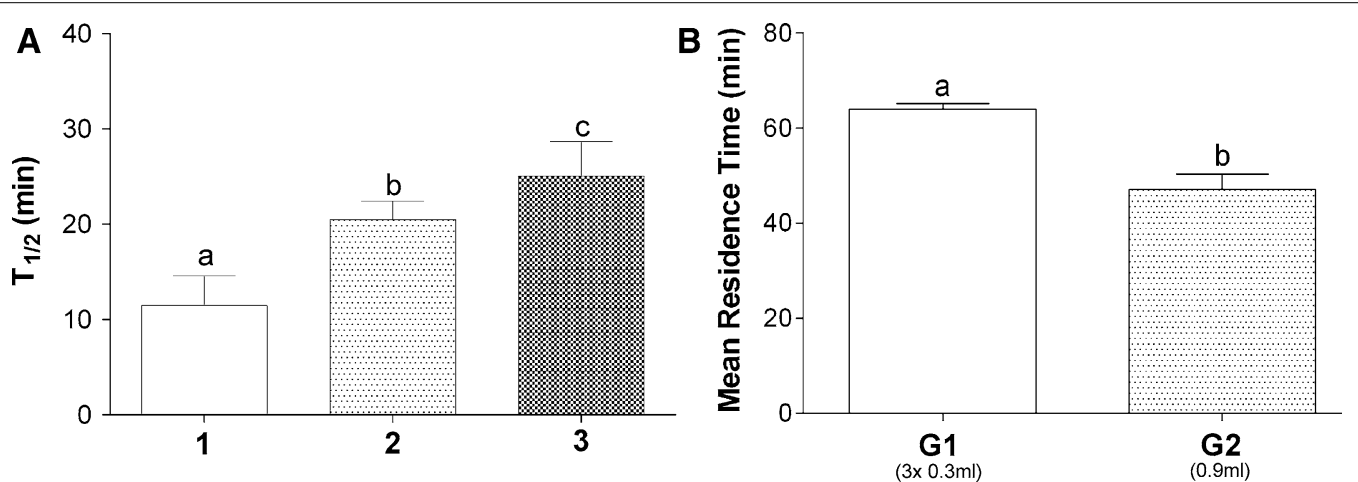

Fig. 4 Magnetic nanoparticle $T_{1 / 2}$. A $T_{1 / 2}$ for each injection in $G 1$. B Comparison of MRT obtained for each group. Different letters indicate significant differences between groups $(p<0.05)$ 
Table 1 MNPs time of arrival in animals' heart

\begin{tabular}{lc}
\hline & $\mathbf{T}_{\mathbf{A}} \mathbf{( s )}$ \\
\hline G1 & \\
1st injection & $21.7 \pm 9.8^{\mathrm{a}}$ \\
2nd injection & $19 \pm 7.6^{\mathrm{a}}$ \\
3rd injection & $18.5 \pm 6.3^{\mathrm{a}}$ \\
G2 & $51.5 \pm 5.73^{\mathrm{b}}$ \\
\hline
\end{tabular}

Different letters indicate significant differences between groups $(p<0.05)$

observed in G2, after the end of injection, may be caused by the greater volume injected, which obviously took a longer interval to arrive at the animals' heart.

\section{Cardiovascular evaluation}

We found a transitory drop in MAP after the MNP injections in all of the animals. In G1, however, we observed a significantly greater pressure variation after the first injection compared with second and third injections, indicating a linear trend $(p<0.0001)$ and suggesting that sequential MNP injections cause smaller modifications of MAP compared with the first injection (Fig. 5A).

We compared arterial pressure before and after each MNP administration. We observed a $44 \pm 4 \%$ decrease after the first injection in the G1 group (Fig. 5A and inset), followed by a $24 \pm 9$ and $22 \pm 9 \%$ decrease after second and third injections, respectively. In the G2 group, the MNP injection caused a $43 \pm 6 \%$ drop in MAP (Fig. 5B, inset). Comparisons of the average pressure drop after the first injection in G1 and G2 revealed no significant difference, indicating that the effects on MAP were not dose-dependent (Fig. 5B).

Table 2 shows a latter MHI after the first injection in the G1 group (77 $\pm 9 \mathrm{~s}$ ), followed by shorter time intervals after second and third injections $(16 \pm 3$ and $17 \pm 6 \mathrm{~s}$, respectively), presenting a significant linear trend $\left(\mathrm{R}^{2}=0.71, p<0.0001\right)$. The same comparison between the sole injection in the G2 group and first injection in the G1 group revealed no significant variation in MHI, also suggesting no MNP dose dependency.

The MAP recovery time was longest for the first injection ( $355 \pm 85 \mathrm{~s}$ ), followed by $41 \pm 34$ and $39 \pm 30 \mathrm{~s}$ for second and third injections, respectively, indicating a significant decreasing linear trend $\left(\mathrm{R}^{2}=0.68, p<0.0001\right)$. The MAP recovery time was not significantly different between the first injection in G1 and sole injection in G2 (Table 2).

The heart rate monitoring, showed slight, but significant, drop in frequency $(p=0.0184$ in G1, $p=0.0003$ in G2). The first MNP injection in G1 caused a $7 \pm 5 \%$ drop in $\mathrm{HR}$, while the MNP injection in G2 caused a $7 \pm 2 \%$ drop in HR. Figure 6A presents the percentage variation in HR after the MNP injections in G1 and G2 groups. All data were compared using $t$ tests, indicating that the first injection in G1 and sole injection in G2 diminished HR, differing significantly from zero value, whereas the second and third injections in the G1 group did not influence HR (Fig. 6A). Also, analysis of the cardiac electrical profile revealed no significant arrhythmia events after the injections nor between first, second, and third injections (Fig. 6B and inset).

\section{Discussion}

In the present study, we employed the ACB system to monitor MNPs in the bloodstream in living animals in real time. Real-time monitoring and in vivo assessment of

Table $2 \mathrm{MHI}$ and MAP recovery time

\begin{tabular}{lcc}
\hline & MHI (s) & MAP recovery time (s) \\
\hline G1 & & \\
1st injection & $77 \pm 9^{\mathrm{a}}$ & $355 \pm 85^{\mathrm{a}}$ \\
2nd injection & $16 \pm 3^{\mathrm{b}}$ & $41 \pm 34^{\mathrm{b}}$ \\
3rd injection & $17 \pm 6^{\mathrm{b}}$ & $39 \pm 30^{\mathrm{b}}$ \\
G2 & $85 \pm 17^{\mathrm{a}}$ & $329 \pm 33^{\mathrm{a}}$ \\
\hline
\end{tabular}

Different letters indicate significant differences between groups $(p<0.05)$
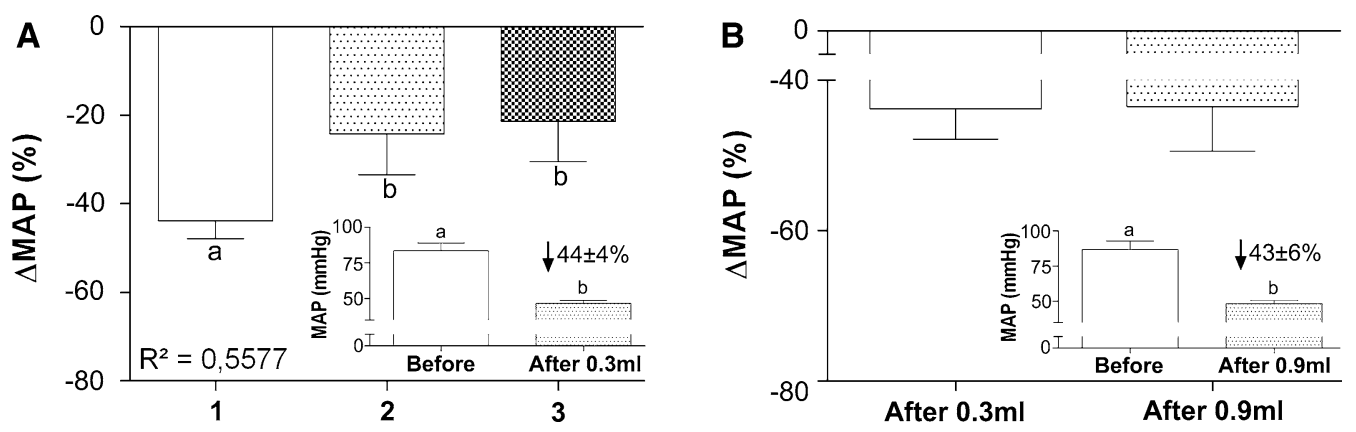

Fig. 5 Effects of MNP administration on arterial pressure. A Percentage of MAP drop after each injection in G1. (Inset) Mean arterial pressure before and after the first injection in G1. B Percentage of MAP drop after the sole injection in G2. (Inset) Mean arterial pressure before and after the injection in G2. Different letters indicate significant differences between groups $(p<0.05)$ 

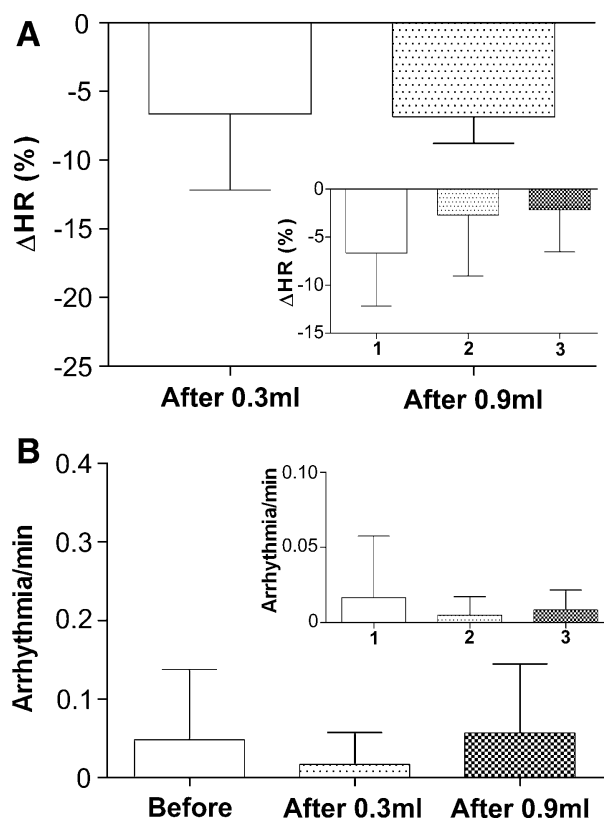

Fig. 6 Percent variation in $\mathrm{HR}$ and quantification of arrhythmia events after MNP administration. A Percent variation in HR measured after the first injection in $\mathrm{G} 1$ and after the sole injection in G2. (Inset) Variation in HR for each injection in G1. B Arrhythmia events quantified before and after the first MNP injection in G1 and after the sole injection in G2. (Inset) number of arrhythmia events for each injection in $\mathrm{G} 1$

nanoparticles remain a challenge that significantly hampers their translational potential [21]. Several approaches have been employed on this task. Table 3 summarized some of these studies, the nanoparticle features and the detection technique employed on the circulation time assessment. Although Table 3 emphasizes the large volume of information and the importance of such studies, most of these techniques cannot be applied to in vivo nor real-time measurements. Thus, the possibility of delivering such features has drawn attention and is a crucial step to towards clinical applications [13, 21, 37].

Our ACB results revealed a similar magnetic intensity curve pattern with regard to particle concentrations in all of the animals and for each MNP administration. Each injection caused a rapid peak signal intensity, which was related to the high blood concentration of magnetic material, followed by exponential decay, associated with the nanoparticle distribution within vascular compartments and probably related with MNPs clearance from the bloodstream (Fig. 2).

The ACB system analysis demonstrated good reproducibility and efficient data acquisition while monitoring circulating MNPs in real time. The reproducibility was verified by comparing the $\mathrm{I}_{\mathrm{INC}}$ measured after each administration in two groups that received same doses of MNPs by different protocols. All of the $\mathrm{I}_{\mathrm{INC}}$ values recorded after each injection in G1 were similar. The sum of $\mathrm{I}_{\mathrm{INC}}$ for all three injections for each animal was not significantly different from the $\mathrm{G} 2$ group, indicating a linear ACB signal response to particle concentrations in the bloodstream.

After the initial ACB signal peak that resulted from the MNP injections, we observed an intensity drop that can be attributed to distribution in the plasmatic compartment and indirectly associated with particle uptake.

Since the final destination of nanoparticles within biological systems is a key aspect of their removal from the bloodstream and thus intrinsically related to circulation time. The main retention and uptake processes are related to specific retention organs and inherent filtration, endocytosis, and metabolic function $[15,20]$. These patterns, however, also depend on the shape, diameter, coating, and surface charge of nanoparticles, which influence uptake and the route of elimination. The average size of the MNPs that were used in the present study was within the 10-100 $\mathrm{nm}$ range. Magnetic nanoparticles of this size pass through discontinuous capillaries and are readily taken up by the reticuloendothelial system, mostly in the liver and spleen [11, 17, 38, 39].

Studies that are based on particle accumulation usually rely on circulation time to deduce uptake by biological processes, such as the EPR effect for tumor treatment [6, $20]$ and angiography or liver imaging [40-42]. Nanoparticles with a short $T_{1 / 2}$ can be used as contrast agents for liver imaging, in which a shorter $\mathrm{T}_{1 / 2}$ decreases the time between nanoparticle administration and image acquisition, thus making the procedure faster and reducing patient discomfort.

Many previous studies have reported the $T_{1 / 2}$ for MNPs, ranging from a few minutes to several hours, depending on particle size and coating material (Table 3). As shown in Table 3, Liu et al. [19] and Huang et al. [20] reported a circulation time of around $2 \mathrm{~h}$ for polyethylene glycol (PEG)-coated nanoparticles, whereas Lacava et al. [18], Lacava et al. [16], and Jain et al. [13] reported a circulation time around $10 \mathrm{~min}$ for dextran-coated nanoparticles. The difference between those nanocarriers might be related to the protein opsonization (Corona) effect [43]. Thus, the nanoparticle circulation time $\left(T_{1 / 2}\right)$ is an intrinsic property, strongly associated with their synthesis process and function.

With regard to the $T_{1 / 2}$ values obtained, the citratecoated MNPs employed in this study were within the average values for particles with similar features. Our results revealed a positive linear relation between sequential MNP administrations and $\mathrm{T}_{1 / 2}$, suggesting a saturation pattern that interfered with the MNP uptake rate, which directly altered the nanoparticle circulation 
Table 3 MNP characteristics, $T_{1 / 2}$, species, dose, method and technique used in the study

\begin{tabular}{|c|c|c|c|c|c|c|}
\hline Core/coat & $\mathrm{CD} / \mathrm{HD}(\mathrm{nm})$ & $T_{1 / 2}$ & Species & Dose & Method/technique & References \\
\hline Maghemite/n.a. & $>40 /$ n.a. & $<10 \min$ & n.a. & n.a. & n.a./n.a. & {$[56]$} \\
\hline $\mathrm{Gd}_{2} \mathrm{O}_{3} / \mathrm{PVP}$ & 2.9/15.7 & $>12 \min$ & Mice & n.a. & In vivo/MRI (7T) $(0.1 \mathrm{~Hz})$ & {$[37]$} \\
\hline Magnetite/dextran & 9.4/n.a. & $10 \min$ & Mice & n.a. & Blood samples/ESR & [18] \\
\hline Ferumoxide/n.a. & $4.3-6.2 / 19$ & $3.7 \mathrm{~h}$ & Rats & $40 \mu \mathrm{mol} \mathrm{Fe} / \mathrm{kg}$ & $\begin{array}{l}\text { Blood samples/gamma } \\
\text { counter }\end{array}$ & [57] \\
\hline $\mathrm{KMnF}_{3} / \mathrm{PEG}-10,000$ & 18-23/n.a. & $1.81 \mathrm{~h}$ & Mice & 20 mg/kg & Blood samples/ICP-MS & [19] \\
\hline Magnetite/PEG-2000 & $11.3 / 23.8$ & $2 \mathrm{~h}$ & Mice & $1.7 \mathrm{~g} \mathrm{Fe} / \mathrm{kg}$ & Blood samples/ICP-MS & {$[20]$} \\
\hline Iron oxide/dextran & $5-15 / 120-180$ & $6-19.8 \min$ & Human & $15 \mu \mathrm{mg} \mathrm{Fe} / \mathrm{kg}$ & n.a./n.a. & [58] \\
\hline Iron oxide/n.a. & n.a./80 & $12.8 \pm 10.3 \mathrm{~min}$ & Human & $10-40 \mu \mathrm{mol} / \mathrm{kg}$ & Blood samples/relaxometry & {$[42]$} \\
\hline $\mathrm{NaYF}_{4}(\mathrm{Yb}: \mathrm{Er}) / \mathrm{PEG}$ & $180 / 220$ & $4.75 \pm 2.2 \mathrm{~min}$ & Mice & $5 \mathrm{mg} / \mathrm{ml}(200 \mu \mathrm{l})$ & Blood samples/ICP-AES & [59] \\
\hline Iron oxide/oleic acid & $15-25 / 210-250$ & $31.2 \mathrm{~min}$ & Mice & $7 \mathrm{mg} \mathrm{Fe} / \mathrm{kg}$ & In vivo/MRI (9.4T) $(0.1 \mathrm{~Hz})$ & [13] \\
\hline Iron oxide/dextran & $5-15 / 140$ & $6.4 \mathrm{~min}$ & Mice & $7 \mathrm{mg} \mathrm{Fe} / \mathrm{kg}$ & In vivo/MRI (9.4T) $(0.1 \mathrm{~Hz})$ & [13] \\
\hline Magnetite/dextran & 9.4/n.a. & $6.9 \pm 0.7 \mathrm{~min}$ & Mice & $\begin{array}{l}4.9 \times 10^{16} \text { particles } / \mathrm{ml} \\
(100 \mu \mathrm{l})\end{array}$ & Blood samples/ESR & {$[16]$} \\
\hline \multirow[t]{5}{*}{ Iron oxide/citrate } & \multirow[t]{5}{*}{$5 / 8$} & $15 \pm 2 \min$ & \multirow[t]{5}{*}{ Rats } & $15 \mu \mathrm{mol} \mathrm{Fe} / \mathrm{kg}$ & \multirow[t]{5}{*}{ In vivo/MRI (1.5T) $(0.01 \mathrm{~Hz})$} & \multirow[t]{5}{*}{ [40] } \\
\hline & & $20 \pm 3 \min$ & & $30 \mu \mathrm{mol} \mathrm{Fe} / \mathrm{kg}$ & & \\
\hline & & $29 \pm 6 \min$ & & $45 \mu \mathrm{mol} \mathrm{Fe} / \mathrm{kg}$ & & \\
\hline & & $37 \pm 5 \min$ & & $60 \mu \mathrm{mol} \mathrm{Fe} / \mathrm{kg}$ & & \\
\hline & & $61 \pm 16 \min$ & & $75 \mu \mathrm{mol} \mathrm{Fe} / \mathrm{kg}$ & & \\
\hline $\begin{array}{l}\text { Magnetite/dimercaptosuc- } \\
\text { cinic acid }\end{array}$ & 12-15/n.a. & $32 \pm 6$ & Rats & 10 mg Fe/kg & Blood samples/ESR & {$[60]$} \\
\hline Iron oxide/dextran & 30/n.a. & $5.8 \mathrm{~h}$ & Mice & $10-20 \mathrm{mg} \mathrm{Fe} / \mathrm{kg}$ & In vivo/PET-CT & {$[61]$} \\
\hline Iron oxide/PEG & $7.1 / 20.3$ & $143 \min$ & Mice & $10 \mathrm{mg} \mathrm{Fe} / \mathrm{kg}$ & In vivo/PET-CT & {$[62]$} \\
\hline
\end{tabular}

n.a. information unavailable in original paper

time. In studies of multiple dosing protocols, once the administration frequency approximates the maximum elimination rate, the pharmacokinetic profile assumes a nonlinear behavior. In this case, the circulation time rises for the following administrations [44]. Since our injections interval were within minutes, considerably below the elimination process starting point, we obtained a nonlinear pharmacokinetic behavior. This behavior was proved by the increasing MNP circulation time obtained for second and third administrations in G1. The first $300 \mu$ injection in G1 resulted in a circulation time of approximately $12 \mathrm{~min}$, while a single $900 \mu \mathrm{l}$ injection in G2 resulted in a $\mathrm{T}_{1 / 2}$ of approximately $47 \mathrm{~min}$, thus confirming the dose-dependent effect on $\mathrm{T}_{1 / 2}$, as described previously [40].

The comparison between the MRT value from all injections in G1 and the MRT from the sole injection in G2 revealed a significant difference, indicating a longer MNP circulation time for sequential administrations. The MRT is an important parameter to quantify the permanence of the drug in the bloodstream following a multiple dosing regimen [33-36]. To the best of our knowledge, there are no studies that evaluated the influence of multiple dosing protocols regarding MNP in the bloodstream, which is the most common protocol for drug administration.
In general, this protocol was used to achieve the steadystate (generally after more than three administrations) [45]. However, here we showed that multiple dosing protocols, even before achieving a steady-state, also prolong the permanence of the MNP in the bloodstream.

The shorter MRT in G2 might be some kind of process initiated by a nanoparticle overdose, which may have triggered specific retention mechanisms that can increase MNP uptake from the bloodstream until stable values were reached. In this way, a protocol with the same dose, divided in three equal amounts, causes a previous saturation in the uptake process that increases the MNP circulation time. Following this rationale, the lack of previous saturation, associated with the rapid particles overload in G2, triggers a higher uptake rate by extravascular compartments, thus promoting a faster MNP clearance from the circulation. Our results suggest a saturated state, which was likely reached because of finite uptake levels by organs. Nonetheless, this difference in MRT between the G1 and G2 groups may help determine the appropriate administration protocol. Our results suggest that fractioned doses result in increased nanoparticles circulation time, while a single MNP dose may result in more circulating particles for a shorter period of time (Figs. 3, 4). This information may be very helpful, since 
most of the studies attempt to improve the MNP circulation time by changing the MNP conjugation and/or coating protocol. Here we demonstrate that changing the administration protocol may be a simple method to achieve this result.

With regard to the nanoparticle $\mathrm{T}_{\mathrm{A}}$, we obtained consistent and reproducible data, with a rise in the signal intensity immediately after the MNP injection, leading to an intensity peak $\left(\mathrm{I}_{\mathrm{INC}}\right)$, which suggests an increase in MNPs blood concentration, followed by a decay profile, indicating the nanoparticles distribution in vascular compartment and its clearance from the circulation. These results, however, might be influenced by specific experimental conditions, such as different routes of administration.

Several studies have investigated the potential application of MNPs as contrast agents for MRI [13, 40, 41] and as heat generators for hyperthermia $[6,11]$. However, few studies have evaluated the cardiovascular effects caused by the MNPs administration. Additionally most of these have been performed using in vitro models and assessed isolated organs and arteries [46-48]. Furthermore, simultaneous assessments of cardiovascular parameters during the injection procedure are an important aspect towards clinical applications.

Our cardiovascular analysis revealed transitory hypotension immediately after the MNP injections in both groups (Fig. 5A, B). The pressure drop observed after the first injection in G1 was statistically similar to G2, indicating that hypotension episodes are not dose-dependent. However, the sequential MNP injection protocol in G1 resulted in different MAP oscillations after each administration.

Mean arterial pressure depends on HR, systolic volume related to contractile cardiac force, and peripheral vascular resistance. We found a slight drop in HR after the first injection in G1 and after the sole injection in G2 (Fig. 6). Although significant, the variation was minimal (7\%) compared with the drop in MAP (44\%), suggesting that the main factor that influenced arterial pressure were reduction in peripheral vascular resistance and/or transitory decrease in cardiac contractility.

The putative reduction in vascular resistance might be caused by shearing stress that results from contacts between MNPs and vessel walls or changes in blood viscosity, which can induce nitric oxide (NO) release by endothelial cells and cause vasodilation and consequent hypotension [49]. Changes in NO levels [50] or bradycardia episodes [46] have been reported after nanoparticle administration. These studies described changes in NO levels that were caused by irreversible and deleterious cellular interactions [49], whereas the drop in HR did not significantly influence arterial pressure [46]. In the present study, we observed transitory and reversible modification of MAP, indicating that other parameters, beyond HR, more intensively influence arterial pressure, while the transitory pattern suggests no damage in the endothelium and in the contractile cardiac capacity. We observed significant differences in episodes of hypotension between the first and subsequent injections in G1 (Table 2), which may be attributable to NO bioavailability or other pressure regulatory elements in the system. Although we found some interesting information, investigating these physiological mechanisms is not the aim of this work and we can assume that more studies are necessary to better elucidate these mechanisms.

The temporary pressure drop observed after MNP administration was reported previously by Iversen et al. [29], who also found that peripheral vascular resistance is the main factor for MAP oscillations. Previous Phase I clinical trials for citrate-coated iron oxide nanoparticles also reported a transitory drop in arterial pressure, in which this parameter returned to normal levels without any intervention [51]. Although MNPs caused significant hypotension, this decrease in arterial pressure was transitory and can be easily controlled by vessel constrictor drugs.

Our assessment of the electrical cardiac profile did not show significant differences between pre- and postinjection (Fig. 6). Acute cardiac arrhythmia is an urgent medical condition, which could limit the intravenous administration of nanoparticles. To our knowledge, very few studies have taken the caution to monitor the electric cardiac profile following intravenous administration of MNP [46]. The data on arrhythmia episodes (Fig. 6B, inset) did not show significant variations for any of the sequential MNP administrations. These data support the general notion that intravenous administration of MNPs does not cause any acute cardiovascular deleterious effects. Since the physiological changes were transitory, easily and rapidly recovered, we can assume that both protocols were safe from the cardiovascular aspect.

Concerning the safety protocols for MNP administration, besides cardiovascular safety, we should draw attention to the Mn doses, since high doses may lead to manganism disease. Manganism is a neurodegenerative disorder and its symptoms are similar to Parkinson's disease [52]. To avoid these problems, the Mn doses have to be lower than the neurotoxicity concentrations [8]. In rats, this dose is believed to be less than $93 \mathrm{mg} / \mathrm{kg}$ [53]. However, some studies indicate higher thresholds for safe protocols, specially regarding partitioned doses $[50,54]$. In our case, we injected a total Mn dose of $32.8 \mathrm{mg} / \mathrm{kg}$, which is very low compared to the dose limit to cause manganism. Also we did not notice any kind of a shortterm manganism symptom, and no animals died during the experiment. 


\section{Conclusions}

Although the present ACB system configuration presents limited spatial resolution compared with gold-standard imaging techniques, such as MRI and MPI, we could successfully assess the circulation time of MNP in the bloodstream. These results indicate that the ACB system may be an important tool for the in vivo monitoring of MNP in real time, which might be combined with other techniques. Furthermore, the ACB system may allow the study of organ perfusion [55], biodistribution and tumor accumulation patterns to define time windows for both diagnostics and treatment modalities based on magnetic nanoparticles.

In summary, we employed an ACB system to detect MNPs in the bloodstream in living animals in real time. By monitoring MNPs in the bloodstream, we were able to determine circulation time in two different MNPs administration protocols. We observed transitory, although significant, cardiovascular alterations after MNP administration. The injections caused similar small and transitory changes in MAP and HR for both groups, without arrhythmia episodes, suggesting that intravenous MNP administration is safe with regard to cardiovascular effects and does not influenced circulation time and accumulation patterns. Aiming clinical applications based on nano-agents, real-time monitoring of these clinical cardiovascular parameters is essential to ensure the protocol safety.

\section{Additional file}

Additional file 1. Development and characterization of the experimental model.

\begin{abstract}
Abbreviations
AC: alternate current; ACB: AC biosusceptometry; Cit-MNP: citrate coated magnetic nanoparticle; CEUA-IBB: Animal Study and Using Committee of Biosciences Institute of Botucatu; DC: direct current; ESR: electron spin resonance; ECG: electrocardiography; G1: group 1; G2: group 2; HR: heart rate; ICP-AES: inductively coupled plasma atomic emission spectroscopy; ICP-MS: inductively coupled plasma mass spectroscopy; $I_{\text {INC: }}$ intensity increase; $I_{\text {MAX: }}$ maximum intensity; $I_{\mathrm{p}}$ : remaining intensity; MAP: mean arterial pressure; $\mathrm{MHI}$ maximum hypotension instant; MNP: magnetic nanoparticle; MPI: magnetic particle imaging; MRI: magnetic resonance imaging; MRT: mean residence time; NO: nitric oxide; PAP: pulsatile arterial pressure; peg: poly-ethylene glycol; PET-CT: positron emission tomography computed tomography; $T_{A}$ : time of arrival in the animal's heart; $T_{1 / 2}$ : half-life in the bloodstream (circulation time).
\end{abstract}

\section{Authors' contributions}

AGP, CCQ, EAZ, FPFM, RVRM and JRAM sensors' design, construction and characterization. FPFM, EAZ, RVRM, AGP and GMM animals' maintenance and preparation for surgical procedure. AGP, PFO and GMM surgical procedures. AGP, PFO, GMM and FPFM data acquisition. AGP, CCQ, RVRM and PFO data quantification. AGP, PFO, CCQ, AFB, NZ, RBO, MFFC and JRAM data interpretation, intellectual input and manuscript preparation. AGP, CCQ and JRAM design conception. AGP, PFO and JRAM design experiments. AFB and NZ magnetic nanoparticles synthesis and characterization. AGP, CCQ, MFFC, AFB and JRAM final version of manuscript and editing. All authors read and approved the final manuscript.

\section{Author details}

${ }^{1}$ Biosciences Institute of Botucatu, São Paulo State University, Botucatu, São Paulo, Brazil. ${ }^{2}$ Physics Institute, Federal University of Goiás, Goiânia, Goiás, Brazil. ${ }^{3}$ Ribeirão Preto School of Medicine, São Paulo University, Ribeirão Prêto, São Paulo, Brazil. ${ }^{4}$ Instituto de Física-Universidade Federal de Goiás, Goiânia, GO 74690-900, Brazil.

\section{Acknowledgements}

We thank Uilian de Andreis for helpful discussions and Juliana F. Matos for her technical support in the early stages of this work.

Funding was provided by Fapesp (Grant No. 2015/14923-9)

\section{Competing interests}

The authors declare that they have no competing interests.

\section{Availability of data and materials}

All data generated or analyzed during this study are included in this published article and its Additional file 1.

\section{Ethics approval}

The authors declare that all of experiments were carried out in accordance with Brazilian legislation regulated by the National Council for the Control of Animal Experimentation (CONCEA) and Ethical Principles in Animal Research formulated by the Brazilian Society of Science in Laboratory Animals, and was approved by the Biosciences Institute/UNESP Ethics Committee on Use of Animals (CEUA) under protocol CEUA-649.

\section{Funding}

This work was supported by Coordenação de Aperfeiçoamento de Pessoal de Nível Superior (CAPES), Fundação de Amparo à Pesquisa do estado de São Paulo (FAPESP), Fundação de Amparo à Pesquisa do estado de Goiás (FAPEG) and Conselho Nacional de Desenvolvimento Científico e Tecnológico (CNPq).

Received: 16 November 2016 Accepted: 10 March 2017

Published online: 21 March 2017

\section{References}

1. Zahn M. Magnetic fluid and nanoparticle applications to nanotechnology. J Nanopart Res. 2001;3:73-8.

2. Lubbe AS, Alexiou C, Bergemann C. Clinical applications of magnetic drug targeting. J Surg Res. 2001;95:200-6.

3. Bulte JWM, Douglas T, Witwer B, Zhang SC, Strable E, Lewis BK, Zywicke H, Miller B, van Gelderen P, Moskowitz BM, et al. Magnetodendrimers allow endosomal magnetic labeling and in vivo tracking of stem cells. Nat Biotechnol. 2001:19:1141-7.

4. Lewin M, Carlesso N, Tung CH, Tang XW, Cory D, Scadden DT, Weissleder $\mathrm{R}$. Tat peptide-derivatized magnetic nanoparticles allow in vivo tracking and recovery of progenitor cells. Nat Biotechnol. 2000;18:410-4.

5. Branquinho LC, Carriao MS, Costa AS, Zufelato N, Sousa MH, Miotto R, Ivkov R, Bakuzis AF. Effect of magnetic dipolar interactions on nanoparticle heating efficiency: implications for cancer hyperthermia. Sci Rep. 2013;3:2887.

6. Rodrigues HF, Mello FM, Branquinho LC, Zufelato N, Silveira-Lacerda EP, Bakuzis AF. Real-time infrared thermography detection of magnetic nanoparticle hyperthermia in a murine model under a non-uniform field configuration. Int J Hyperth. 2013;29:752-67.

7. Kettering M, Winter J, Zeisberger M, Bremer-Streck S, Oehring H, Bergemann C, Alexiou C, Hergt R, Halbhuber KJ, Kaiser WA, Hilger I. Magnetic nanoparticles as bimodal tools in magnetically induced labelling and magnetic heating of tumour cells: an in vitro study. Nanotechnology. 2007;18:175101.

8. Sadighian S, Rostamizadeh K, Hosseini-Monfared H, Hamidi M. Doxorubicin-conjugated core-shell magnetite nanoparticles as dual-targeting carriers for anticancer drug delivery. Colloids Surf B Biointerfaces. 2014;117:406-13.

9. Shubayev VI, Pisanic TR 2nd, Jin S. Magnetic nanoparticles for theragnostics. Adv Drug Deliv Rev. 2009:61:467-77. 
10. Singh A, Sahoo SK. Magnetic nanoparticles: a novel platform for cancer theranostics. Drug Discov Today. 2014;19:474-81.

11. Sun C, Lee JS, Zhang M. Magnetic nanoparticles in MR imaging and drug delivery. Adv Drug Deliv Rev. 2008;60:1252-65.

12. Albanese A, Tang PS, Chan WC. The effect of nanoparticle size, shape, and surface chemistry on biological systems. Annu Rev Biomed Eng. 2012;14:1-16.

13. Jain TK, Richey J, Strand M, Leslie-Pelecky DL, Flask CA, Labhasetwar V. Magnetic nanoparticles with dual functional properties: drug delivery and magnetic resonance imaging. Biomaterials. 2008:29:4012-21.

14. Larsen EK, Nielsen T, Wittenborn T, Rydtoft LM, Lokanathan AR, Hansen L, Ostergaard L, Kingshott P, Howard KA, Besenbacher F, et al. Accumulation of magnetic iron oxide nanoparticles coated with variably sized polyethylene glycol in murine tumors. Nanoscale. 2012;4:2352-61.

15. Yu M, Zheng J. Clearance pathways and tumor targeting of imaging nanoparticles. ACS Nano. 2015;9:6655-74.

16. Lacava LM, Lacava ZGM, Da Silva MF, Silva O, Chaves SB, Azevedo RB, Pelegrini F, Gansau C, Buske N, Sabolovic D, Morais PC. Magnetic resonance of a dextran-coated magnetic fluid intravenously administered in mice. Biophys J. 2001;80:2483-6.

17. Estevanato LL, Lacava LM, Carvalho LC, Azevedo RB, Silva O, Pelegrini F, Bao SN, Morais PC, Lacava ZG. Long-term biodistribution and biocompatibility investigation of dextran-coated magnetite nanoparticle using mice as the animal model. J Biomed Nanotechnol. 2012;8:301-8.

18. Lacava LM, Lacava ZGM, Azevedo RB, Chaves SB, Garcia VAP, Silva O, Pelegrini F, Buske N, Gansau C, Da Silva MF, Morais PC. Use of magnetic resonance to study biodistribution of dextran-coated magnetic fluid intravenously administered in mice. J Magn Magn Mater. 2002;252:367-9.

19. Liu ZJ, Song XX, Xu XZ, Tang Q. Biocompatible KMnF3 nanoparticular contrast agent with proper plasma retention time for in vivo magnetic resonance imaging. Nanotechnology. 2014;25:155101.

20. Huang HS, Hainfeld JF. Intravenous magnetic nanoparticle cancer hyperthermia. Int J Nanomedicine. 2013;8:2521-32.

21. Sanhai WR, Sakamoto JH, Canady R, Ferrari M. Seven challenges for nanomedicine. Nat Nanotechnol. 2008;3:242-4.

22. Weizenecker J, Gleich B, Rahmer J, Dahnke H, Borgert J. Three-dimensional real-time in vivo magnetic particle imaging. Phys Med Biol. 2009;54:L1-10.

23. Zheng B, Vazin T, Goodwill PW, Conway A, Verma A, Ulku Saritas E, Schaffer D, Conolly SM. Magnetic particle imaging tracks the long-term fate of in vivo neural cell implants with high image contrast. Sci Rep. 2015;5:14055

24. Miranda JRA, Oliveira RB, Sousa PL, Braga FJH, Baffa O. A novel biomagnetic method to study gastric antral contractions. Phys Med Biol. 1997:42:1791-9.

25. Cora A, Andreis U, Romeiro FG, Americo MF, Oliveira RB, Baffa O, Miranda JRA. Magnetic images of the disintegration process of tablets in the human stomach by ac biosusceptometry. Phys Med Biol. 2005:50:5523-34.

26. Andreis U, Americo MF, Cora LA, Oliveira RB, Baffa O, Miranda JR. Gastric motility evaluated by electrogastrography and alternating current biosusceptometry in dogs. Physiol Meas. 2008;29:1023-31.

27. Quini CC, Americo MF, Cora LA, Calabresi MF, Alvarez M, Oliveira RB, Miranda JR. Employment of a noninvasive magnetic method for evaluation of gastrointestinal transit in rats. J Biol Eng. 2012;6:6.

28. Nunes AD, Ramalho LS, Souza AP, Mendes EP, Colugnati DB, Zufelato N, Sousa MH, Bakuzis AF, Castro CH. Manganese ferrite-based nanoparticles induce ex vivo, but not in vivo, cardiovascular effects. Int J Nanomedicine. 2014;9:3299-312.

29. Iversen NK, Frische S, Thomsen K, Laustsen C, Pedersen M, Hansen PB, Bie P, Fresnais J, Berret JF, Baatrup E, Wang T. Superparamagnetic iron oxide polyacrylic acid coated gamma-Fe2O3 nanoparticles do not affect kidney function but cause acute effect on the cardiovascular function in healthy mice. Toxicol Appl Pharmacol. 2013;266:276-88.

30. Hoar EF, Meister ML: Distributed resistance leadwire harness assembly for physiological monitoring during magnetic resonance imaging. Google Patents; 2000.

31. Itri R, Depeyrot J, Tourinho FA, Sousa MH. Nanoparticle chain-like formation in electrical double-layered magnetic fluids evidenced by smallangle X-ray scattering. Eur Phys J E. 2001;4:201-8.
32. McNeeley KM, Annapragada A, Bellamkonda RV. Decreased circulation time offsets increased efficacy of PEGylated nanocarriers targeting folate receptors of glioma. Nanotechnology. 2007;18:385101.

33. Yamaoka K, Nakagawa T, Uno T. Statistical moments in pharmacokinetics. J Pharmacokinet Biopharm. 1978;6:547-58.

34. Sahin S, Benet LZ. The operational multiple dosing half-life: a key to defining drug accumulation in patients and to designing extended release dosage forms. Pharm Res. 2008;25:2869-77.

35. Wagner JG. Dosage intervals based on mean residence times. J Pharm Sci. 1987;76:35-8

36. Podczeck F, Newton JM, Yuen KH. The description of the gastrointestinal transit of pellets assessed by gamma scintigraphy using statistical moments. Pharm Res. 1995;12:376-9.

37. Fang J, Chandrasekharan P, Liu XL, Yang Y, Lv YB, Yang CT, Ding J. Manipulating the surface coating of ultra-small Gd2O3 nanoparticles for improved T1-weighted MR imaging. Biomaterials. 2014;35:1636-42.

38. Gamarra LF, daCosta-Filho AJ, Mamani JB, de Cassia Ruiz R, Pavon LF, Sibov TT, Vieira ED, Silva AC, Pontuschka WM, Amaro E Jr. Ferromagnetic resonance for the quantification of superparamagnetic iron oxide nanoparticles in biological materials. Int J Nanomedicine. 2010;5:203-11.

39. Choi HS, Liu W, Misra P, Tanaka E, Zimmer JP, Itty Ipe B, Bawendi MG, Frangioni JV. Renal clearance of quantum dots. Nat Biotechnol. 2007; 25:1165-70.

40. Taupitz M, Schnorr J, Abramjuk C, Wagner S, Pilgrimm H, Hunigen $\mathrm{H}$, Hamm B. New generation of monomer-stabilized very small superparamagnetic iron oxide particles (VSOP) as contrast medium for MR angiography: preclinical results in rats and rabbits. J Magn Reson Imaging. 2000;12:905-11.

41. Wagner S, Schnorr J, Pilgrimm H, Hamm B, Taupitz M. Monomer-coated very small superparamagnetic iron oxide particles as contrast medium for magnetic resonance imaging - preclinical in vivo characterization. Invest Radiol. 2002;37:167-77.

42. Stark DD, Weissleder R, Elizondo G, Hahn PF, Saini S, Todd LE, Wittenberg J, Ferrucci JT. Superparamagnetic iron-oxide-clinical-application as a contrast agent for MR imaging of the liver. Radiology. 1988;168:297-301.

43. Monopoli MP, Aberg C, Salvati A, Dawson KA. Biomolecular coronas provide the biological identity of nanosized materials. Nat Nanotechnol. 2012;7:779-86.

44. Goodman LS, Gilman A, Brunton LL. Goodman \& Gilman's manual of pharmacology and therapeutics. New York: McGraw-Hill Medical; 2008.

45. Charrois GJ, Allen TM. Multiple injections of pegylated liposomal Doxorubicin: pharmacokinetics and therapeutic activity. J Pharmacol Exp Ther. 2003;306:1058-67.

46. Apte K, Hede S. Cardiovascular effects of cetyl trimethyl ammonium bromide-protected gold nanoparticles. Indian J Pharmacol. 2007:39:210-3.

47. Stampfl A, Maier M, Radykewicz R, Reitmeir P, Gottlicher M, Niessner R. Langendorff heart: a model system to study cardiovascular effects of engineered nanoparticles. ACS Nano. 2011;5:5345-53.

48. Thompson LC, Frasier CR, Sloan RC, Mann EE, Harrison BS, Brown JM, Brown DA, Wingard CJ. Pulmonary instillation of multi-walled carbon nanotubes promotes coronary vasoconstriction and exacerbates injury in isolated hearts. Nanotoxicology. 2014;8:38-49.

49. Rosas-Hernandez H, Jimenez-Badillo S, Martinez-Cuevas PP, Gracia-Espino E, Terrones H, Terrones M, Hussain SM, Ali SF, Gonzalez C. Effects of 45-nm silver nanoparticles on coronary endothelial cells and isolated rat aortic rings. Toxicol Lett. 2009;191:305-13.

50. Bock NA, Paiva FF, Silva AC. Fractionated manganese-enhanced MRI. NMR Biomed. 2008;21:473-8.

51. Taupitz M, Wagner S, Schnorr J. Phase I clinical evaluation of citratecoated monocrystalline very small superparamagnetic iron oxide particles as a new contrast medium for magnetic resonance imaging. Invest Radiol. 2004;39:625.

52. O'Neal SL, Zheng W. Manganese toxicity upon overexposure: a decade in review. Curr Environ Health Rep. 2015;2:315-28.

53. Silva AC, Bock NA. Manganese-enhanced MRI: an exceptional tool in translational neuroimaging. Schizophr Bull. 2008;34:595-604.

54. Zhang S, Zhou Z, Fu J. Effect of manganese chloride exposure on liver and brain mitochondria function in rats. Environ Res. 2003;93:149-57.

55. Quini CC, Matos JF, Próspero AG, Calabresi MFF, Zufelato N, Bakuzis AF, Baffa O, Miranda JRA. Renal perfusion evaluation by alternating current 
biosusceptometry of magnetic nanoparticles. J Magn Magn Mater. 2015;380:2-6. doi:10.1016/j.jmmm.2014.09.073.

56. Duguet E, Vasseur S, Mornet S, Devoisselle JM. Magnetic nanoparticles and their applications in medicine. Nanomedicine. 2006;1:157-68.

57. Bengele HH, Palmacci S, Rogers J, Jung CW, Crenshaw J, Josephson L. Biodistribution of an ultrasmall superparamagnetic iron oxide colloid, BMS 180549, by different routes of administration. Magn Reson Imaging 1994;12:433-42.

58. Liu JP. Nanoscale magnetic materials and applications. New York: Springer Verlag; 2009.

59. Cheng L, Yang K, Li YG, Zeng X, Shao MW, Lee ST, Liu Z. Multifunctional nanoparticles for upconversion luminescence/MR multimodal imaging and magnetically targeted photothermal therapy. Biomaterials. 2012;33:2215-22.
60. Gobbo OL, Wetterling F, Vaes P, Teughels S, Markos F, Edge D, Shortt CM, Crosbie-Staunton K, Radomski MW, Volkov Y, Prina-Mello A. Biodistribution and pharmacokinetic studies of SPION using particle electron paramagnetic resonance, MRI and ICP-MS. Nanomedicine. 2015;10:1751-60.

61. Devaraj NK, Keliher EJ, Thurber GM, Nahrendorf M, Weissleder R. F-18 labeled nanoparticles for in vivo PET-CT imaging. Bioconjug Chem. 2009:20:397-401.

62. Glaus C, Rossin R, Welch MJ, Bao G. In vivo evaluation of Cu-64-labeled magnetic nanoparticles as a dual-modality PET/MR imaging agent. Bioconjug Chem. 2010;21:715-22.

\section{Submit your next manuscript to BioMed Central and we will help you at every step:}

- We accept pre-submission inquiries

- Our selector tool helps you to find the most relevant journal

- We provide round the clock customer support

- Convenient online submission

- Thorough peer review

- Inclusion in PubMed and all major indexing services

- Maximum visibility for your research

Submit your manuscript at www.biomedcentral.com/submit 